\title{
SIMULTANEOUS ESTIMATION OF CURCUMIN AND GEFITINIB IN BULK BY USING RP-HPLC TECHNIQUE WITH PDA DETECTOR
}

\section{SAGAR KISHOR SAVALE}

Department of Pharmaceutics, R. C. Patel Institute of Pharmaceutical Education and Research, Shirpur 425-405, MS, India.

\begin{tabular}{ll}
\hline & ABSTRACT \\
& Plan: RP-HPLC method development and validation for the simultaneous \\
& estimation of curcumin and Gefitinib in bulk. \\
& Preface: RP-HPLC chromatographic methods have been widely employed in \\
Key words: Isocratic, Curcumin, RP-HPLC, & determination of individual components in a mixture or fixed dose combination. \\
Gefitinib, Validation, & For the ternary mixture containing Curcumin and Gefitinib, no \\
Simultaneous estimation. & chromatographic method for simultaneous evaluation has been reported so far. \\
Correspondence & Thus our aim is to develop a simultaneous estimation method for curcumin and \\
SAGAR KISHOR SAVALE, M.Pharm & gefitinib by using an RP-HPLC method using a PDA detector. \\
Department of Pharmaceutics, & Methodology: The method was validated as per ICH guidelines. The recovery \\
R. C. Patel Institute of Pharmaceutical & studies confirmed the accuracy and precision of the method. \\
Education \& Research, Shirpur, 425405, & Outcome: The proposed method was found to be accurate, repeatability and \\
dist. Dhule, Maharashtra, India. & consistent. It was successfully applied for the analysis of the drug in marketed \\
Mobile: +91 9960885333 & formulation and could be effectively used for the routine analysis of formulation \\
Received: 30 September 2017, & containing the drug without any alteration in the chromatography conditions. \\
Revised: 12 January 2018 &
\end{tabular}

\section{INTRODUCTION}

((1E, 6E)-1, 7-Bis (4-hydroxy-3-methoxyphenyl)-1, 6 heptadiene-3, 5-dione), a polyphenol (Curcumin: CRM) known as diferuloylmethane, has been extensively studied for its therapeutic efficacy for many disorders including Alzheimer, and brain cancer or other CNS disorder, 2, ${ }^{3}$.Curcumin was anticancer drug to inhibit process of angiogenesis, it is process for formation of new blood cells in blood vessel that can responsible for proliferation and uncontrolled growth of tumour cells. It is assumed that, Curcumin (CRM) inhibits that angiogenesis and stops the growth of cancer cells and the CRM-induced apoptosis through down-regulation of HSP 90 protein expression to stop proliferation of tumour cells ${ }^{4,5}$. 
(N(3chlorofluorophenyl)7methoxy6(3morpholinopropoxy) quinazolin-4- amine) is a type of drug (Gefitinib: GFT) called a tyrosine kinase inhibitor (TKI), also known as a cancer growth inhibitor. Gefitinib inhibits the activity of EGFR and VGFR tyrosine kinase receptor, to stop growth of cancer calls ${ }^{6}$.Curcumin enhances gefitinib induced cytotoxicity via down regulation of nuclear factor (NF)$\mathrm{\kappa B}$ and the Akt pathways, thereby reversing MDR. This study aimed to develop a simple, rapid and sensitive method for simultaneous determination analyte (curcumin and gefitinib) in bulk using RPHPLC method. The developed method was successfully applied for the analysis of the CRM and GFT in bulk and Pharmaceutical dosage forms.

\section{EXPERIMENTAL}

\subsection{Chemicals and reagents}

Curcumin (CRM) supplied as a gift sample by Sun pure Extracts Pvt. Ltd (Delhi, India) and Gefitinib (GFT) supplied as a gift sample by Khandelwal industries Pvt. Ltd (Mumbai, India) both drug was used as working standard. All the chemicals used of HPLC Grade (MERCK. Chem. Ltd., Mumbai) and double distilled water was used for mobile phase preparation.

\subsection{Instrumentation}

Analyses were carried out using an Agilent 1200 HPLC system (Agilent technologies, USA). 1200 HPLC system was equipped with quaternary pump and photo diode-array (PDA) detector. All data were acquired and processed using EZ chrome elite software.

\subsection{Chromatographic conditions}

Chromatographic separation was performed by using C-18 column (Qualisil BDS C18, $250 \mathrm{~mm} x$ $4.6 \mathrm{~mm}$ I.D.) coupled with a guard column. Isocratic elution was performed with acetonitrile: water with $0.1 \%$ formic acid $(30: 70 \mathrm{v} / \mathrm{v})$ at a flow rate of $0.2 \mathrm{~mL} / \mathrm{min}$. The mobile phase was selected to give proper resolution of peaks.

\subsection{Preparation of standard solutions and quality control $(Q C)$ samples}

\subsubsection{Preparation of standard solutions of CRM}

Certified reference standards of CRM were weighed $100 \mathrm{mg}$ accurately and transferred into a $100 \mathrm{ml}$ of volumetric flask and dissolved in $100 \mathrm{ml}$ of methanol to obtain a solution having concentration1000 $\mu \mathrm{g} / \mathrm{mL}$ solution. The working standard solutions were $10-60 \mu \mathrm{g} / \mathrm{mL}$ concentrations.

\subsubsection{Preparation of standard solutions of GFT}

Certified reference standards of GFT were weighed accurately and transferred $100 \mathrm{mg}$ accurately and transferred into a $100 \mathrm{ml}$ of volumetric flask and dissolved in $100 \mathrm{ml}$ of methanol to obtain a solution having concentration $1000 \mu \mathrm{g} / \mathrm{mL}$ solution. The working standard solutions were of $10-60$ $\mu \mathrm{g} / \mathrm{mL}$ concentrations. 


\subsection{Method development}

Method development was important to judge the quality, reliability and consistency of analytical results. It is the process for proving that analytical method is acceptable for determination of the concentration of drugs. The final chromatographic condition for method development was reported in Table 1.

Table 1. Final Chromatographic Conditions

\begin{tabular}{cc}
\hline Chromatographic Mode & Chromatographic Condition \\
\hline Standard solution & For Bulk: $100 \mu \mathrm{g} / \mathrm{mL}$ solution in methanol \\
HPLC System & Agilent Technologies HPLC system \\
Pump & Reciprocating Quaternary pump \\
Detector & Photo Diode Array Detector \\
Data processor & EZ Chrome Elite Chromatographic data system \\
Stationary phase & Qualisil BDS C $18,250 \mathrm{~mm} \times 4.6 \mathrm{~mm} \mathrm{I.D.}$ \\
Mobile phase & Acetonitrile: water with $0.1 \%$ formic acid $(30: 70 \mathrm{v} / \mathrm{v})$ \\
Detection wavelength & $242 \mathrm{~nm}$ \\
Flow rate & $0.2 \mathrm{ml} / \mathrm{min}$ \\
Sample size & $20 \mu 1$ \\
\hline
\end{tabular}

\subsection{Method validation}

Application of the proposed method to bulk sample, linearity, recovery, precision, repeatability, ruggedness, robustness and sensitivity were determined in method validation. The linearity of an analytical method is its ability to elicit test results that are directly, or by a well-defined mathematical transformation, proportional to the concentration of analyte in sample within a given range.

Percent recovery of the proposed method was ascertained on the basis of recovery studies performed by standard addition method. The percent recovery as well as average percent recovery was calculated. Recovery should be assessed using minimum 9 determinations over minimum 3 concentrations level covering specified range. Recovery study was performed three different level $80 \%, 100 \%$ and $120 \%$.

The precision is the measure of either the degree of reproducibility or repeatability of analytical method. It provides an indication of random error. Precision is the measure of how close the data values are to each other for a number of measurements under the same analytical conditions. Intra-day precision was determined by analysing, the three different concentrations $20 \mathrm{mg} / \mathrm{ml}, 30 \mathrm{mg} / \mathrm{ml}$ and 40 $\mathrm{mg} / \mathrm{ml}$ for three times in the same day and Inter-day variability was assessed using above mentioned three concentrations of samples were analysed by three different days, over a period of one week.

Repeatability is measured by multiple time analysis of a homogenous sample of $10 \mu \mathrm{g} / \mathrm{ml}$ solution containing CRM and GFT that indicates the performance of the HPLC instrument under chromatographic conditions.

The ruggedness of the method was determined by carrying out the experiment on different instruments by different operators using different columns of similar types. From stock solution, sample solution containing CRM and GFT $(10 \mu \mathrm{g} / \mathrm{ml})$ was prepared and analyzed by two different analysts using similar operational and environmental conditions. Peak area was measured for same concentration of solutions, three times. 
Robustness of the method was determined by making slight changes in the chromatographic conditions like change in $\mathrm{pH}$ and change in mobile phase ratio. To evaluate robustness few parameters were deliberately varied.

Sensitivity refers to the smallest quantity that can be accurately measured. It also indicates the capacity of the method to measure small variations in concentration. Sensitivity of the proposed method was estimated in terms of Limit of Detection (LOD) and Limit of Quantitation (LOQ). For sample analysis six different concentration range $10-60 \mu \mathrm{g} / \mathrm{ml}$. The linear regression equation of the calibration curve was used to determine the LOD and LOQ.

\section{RESULTS AND DISCUSSION}

\subsection{Method development}

Operating conditions of HPLC, such as component of mobile phase and elution, type of column, were carefully optimized. Different mobile phase compositions were tried, one which included acetonitrile and water (0.1\% ammonia) (40: $60 \% \mathrm{v} / \mathrm{v})$, Acetonitrile: water (0.1\% ammonia) (50: $50 \%$ $\mathrm{v} / \mathrm{v})$, then acetonitrile: water $(0.1 \%$ ammonia) $(20: 80 \% \mathrm{v} / \mathrm{v})$ tried did not give adequate resolution. Mobile phase consisting of Acetonitrile: water with $0.1 \%$ formic acid in the ratio of $(30: 70 \% \mathrm{v} / \mathrm{v})$ gave proper resolution of the two drugs. This method showed the best peak shape and ideal detection response. Furthermore, strong organic solvent in the reversed-phase chromatography can reduce static retention and shorten analysis time. In addition, with the column, all the reference standards (CRM and GFT) can be completely separated with narrow peaks, high sensitivity and no obvious tailing. Sample preparation is extremely important to the whole method in order to reduce possible interference from the sample matrix and increase sensitivity. Typical chromatograms of CRM, GFT, CRM + GFT and CRM-GFT in laboratory mixture were shown in Figure 1.
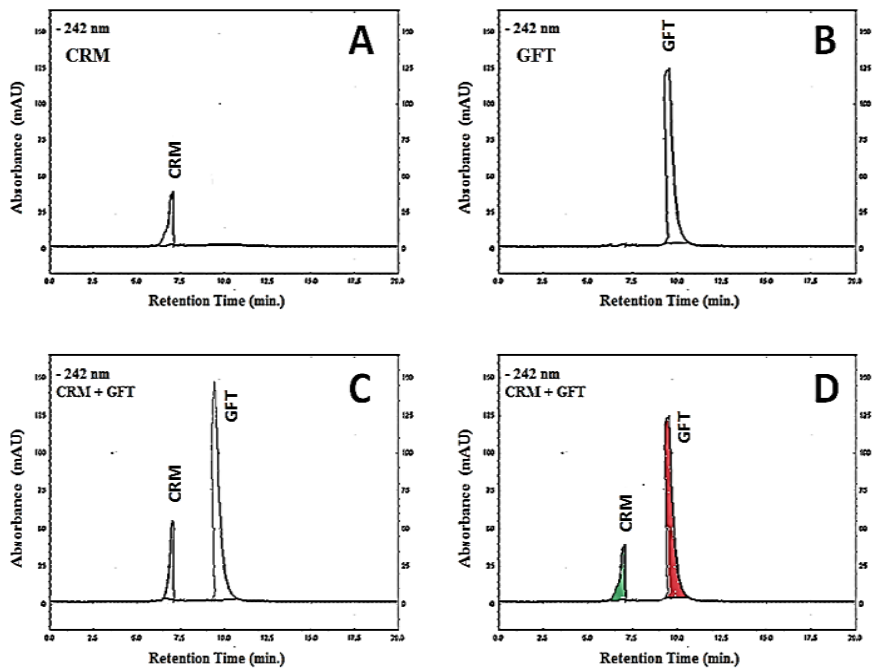

Figure 1. HPLC chromatogram: Chromatogram of curcumin (CRM) (A), Chromatogram of gefitinib (B), Chromatogram of curcumin + gefitinib (CRM + GFT) (C), Chromatogram of bulk (CRM + GFT) in laboratory mixture (D) 


\subsection{Method validation}

\subsubsection{Linearity}

The linearity concentration was in the range of $10-60 \mu \mathrm{g} / \mathrm{mL}$ for CRM and GFT (Figure 2). The correlation coefficients $\left(\mathrm{R}^{2}\right)$ forCRM was 0.999 and GFT was 0.9993.
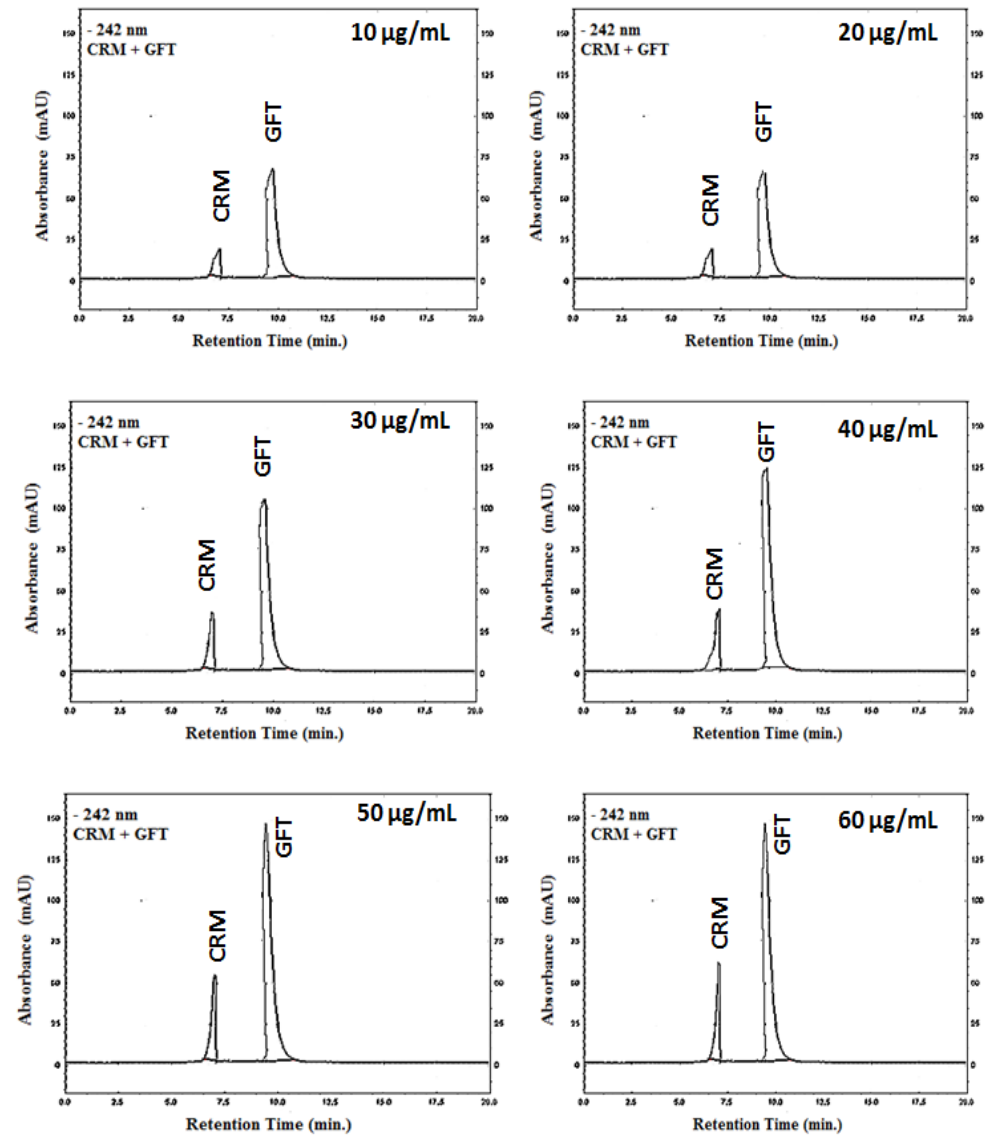

Figure 2. Linearity study of CRM and GFT: chromatogram having concentration range 10-60 $\mu \mathrm{g} / \mathrm{mL}$

\subsubsection{Application of the proposed method to bulk sample}

Bulk sample was determined chromatographic standards in laboratory mixture and the concentration of drug was determined from their respective linearity curves and Results are shown in Table 2.

\subsubsection{Recovery study}

The recovery of an analytical procedure expresses the closeness of agreement between the value which is accepted either as a conventional true value or an accepted reference value and the value found. Recovery studies of proposed method were carried out, respective data is obtained and mentioned in Table 2. Recovery study was determined at three levels $80 \%, 100 \%, 120 \%$ at each level three determinations were performed. 


\subsubsection{Precision and Repeatability}

Intra-day and Inter-day precision sample analysis results were reported in Table 2. The $\%$ RSD for CRM and GFT was less than $2.0 \%$. The results are showing that the proposed method was precise. Repeatability expresses the precision under the same operating conditions over a short interval of time. Results of repeatability were reported in Table 2.

Table 2. Bulk Sample, Recovery, Presion and Repeatability

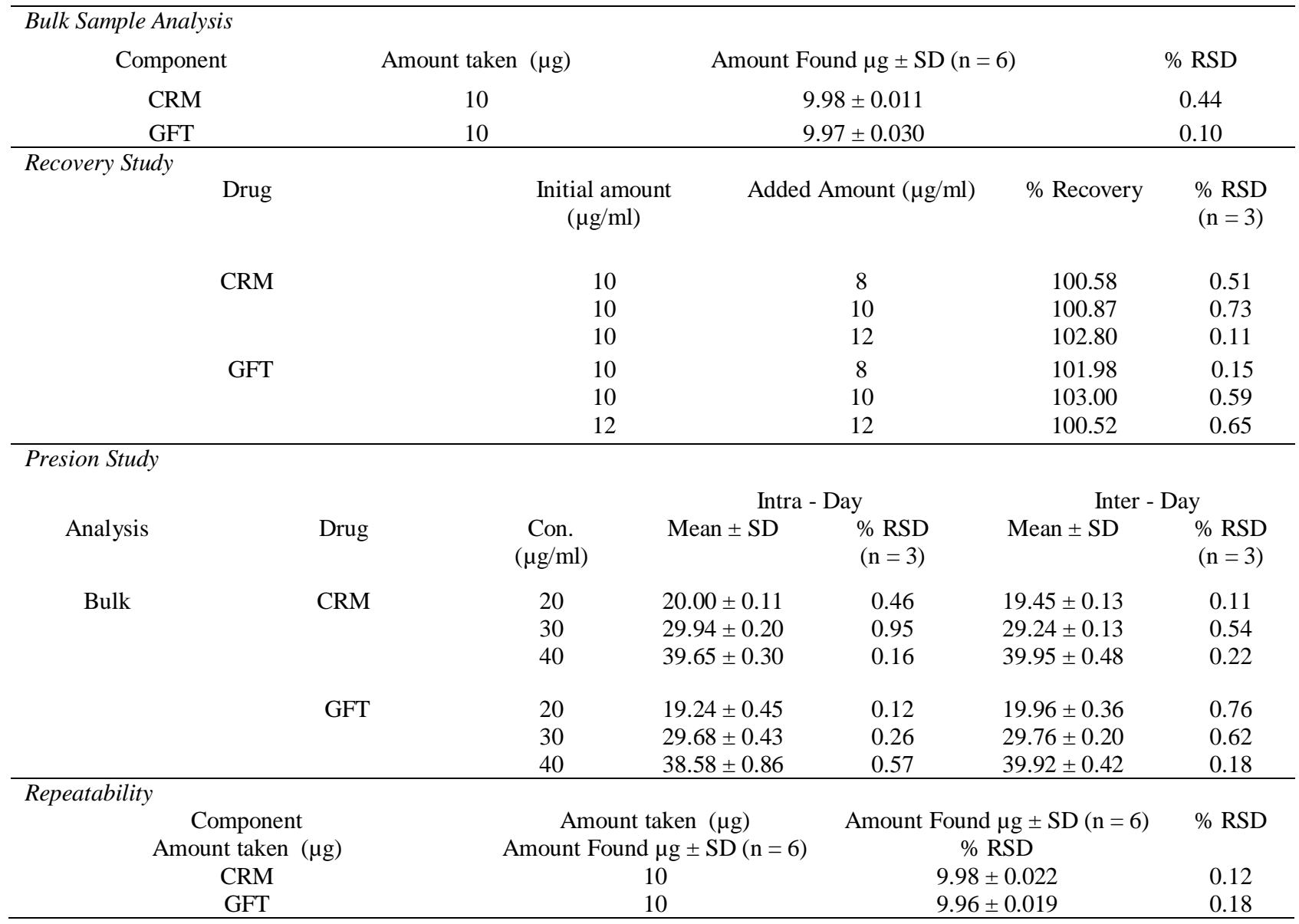

\subsubsection{Ruggedness, Robustness and sensitivity}

Ruggedness of analytical method is the degree of reproducibility of test results obtained by the analysis of the same samples under a variety of conditions such different instruments, different analysts. It was observed that there were no marked changes in the chromatograms, which demonstrated that the HPLC method developed was rugged. Robustness is the measure of the capacity of the analytical method to remain unaffected by Small but deliberate variations in procedure, it was observed that there were no marked changes in the chromatograms, which demonstrated that the HPLC method developed was robust. 
Sensitivity of the proposed method was estimated in terms of Limit of Detection (LOD) and Limit of Quantitation (LOQ). The linear regression equation of the calibration curve was used to determine the LOD and LOQ. Result of ruggedness, robustness and sensitivity (LOD and LOQ) studies were reported in Table 3.

Table 3. Ruggedness, Robustness and Sensitivity Study

\begin{tabular}{|c|c|c|c|}
\hline \multicolumn{4}{|l|}{ Ruggedness } \\
\hline & $\%$ Amount Found & \multicolumn{2}{|c|}{$\% \operatorname{RSD}(\mathrm{n}=3)$} \\
\hline Drug & Analyst II & Analyst I & Analyst II \\
\hline CRM & 100.54 & 0.44 & 0.16 \\
\hline GFT & 100.44 & 0.24 & 0.46 \\
\hline \multicolumn{4}{|l|}{ Robustness } \\
\hline \multicolumn{4}{|c|}{ Chromatographic conditions (change in Mobile Phase) } \\
\hline \multicolumn{4}{|c|}{ Acetonitrile : Water with $1.0 \%$ formic acid $(20: 80)$} \\
\hline Conc. $(\mu \mathrm{g} / \mathrm{ml})$ & Retention time (CRM) & \multicolumn{2}{|c|}{ Retention time (GFT) } \\
\hline 10 & 9.12 & \multicolumn{2}{|c|}{7.32} \\
\hline 10 & 9.23 & \multicolumn{2}{|c|}{7.37} \\
\hline 10 & 9.54 & \multicolumn{2}{|c|}{7.26} \\
\hline \multicolumn{4}{|c|}{ Acetonitrile : Water with $1.0 \%$ formic acid (40:60) } \\
\hline 10 & 9.38 & \multicolumn{2}{|c|}{7.35} \\
\hline 10 & 9.36 & \multicolumn{2}{|c|}{7.25} \\
\hline 10 & 9.52 & \multicolumn{2}{|c|}{7.36} \\
\hline \multicolumn{4}{|c|}{ Chromatographic conditions (change in $\mathrm{pH}$ ) } \\
\hline \multicolumn{4}{|c|}{1.19} \\
\hline 10 & 9.41 & \multicolumn{2}{|c|}{7.39} \\
\hline 10 & 9.45 & \multicolumn{2}{|c|}{7.35} \\
\hline 10 & 9.55 & \multicolumn{2}{|c|}{7.38} \\
\hline \multicolumn{4}{|c|}{5.42} \\
\hline 10 & 9.37 & \multicolumn{2}{|c|}{7.39} \\
\hline 10 & 9.55 & \multicolumn{2}{|c|}{7.22} \\
\hline 10 & 9.53 & \multicolumn{2}{|c|}{7.37} \\
\hline \multicolumn{4}{|l|}{ Sensitivity } \\
\hline Drug & LOD & \multicolumn{2}{|c|}{ LOQ } \\
\hline CRM & $0.38 \pm 0.04$ & \multicolumn{2}{|c|}{$0.95 \pm 0.10$} \\
\hline GFT & $0.36 \pm 0.02$ & \multicolumn{2}{|c|}{$0.93 \pm 0.12$} \\
\hline
\end{tabular}

\section{CONCLUSION}

In this validation of analytical method for simultaneous estimation for CRM and GFT quantification evaluated for linearity, precision, recovery, repeatability, ruggedness, robustness and sensitivity (LOD-LOQ) in order to establish the suitability of analytical method. The method is established by caring out simultaneous analysis of analyte and the low value of \% RSD showed that the method is precise within the acceptance limit of $2 \%$. The method is validated in compliance with ICH guidelines is suitable for simultaneous estimation of CRM and GFT with excellent linearity, recovery and precision. 


\section{References}

1. Andriamanana I, Gana I, Duretz B, Hulin A. Simultaneous analysis of anticancer agents bortezomib, imatinib, nilotinib, dasatinib, erlotinib, lapatinib, sorafenib, sunitinib and vandetanib in human plasma using LC/MS/MS. Journal of Chromatography B 2013; 926: 83-91.CrossRef PMid:23562906

2. Couchman L, Birch M, Ireland R, Corrigan A, Wickramasinghe S, Josephs D. An automated method for the measurement of a range of tyrosine kinase inhibitors in human plasma or serum using turbulent flow liquid chromatography-tandem mass spectrometry. Anal Bioanal Chem.2012; 403: 1685-1695. CrossRef PMid:22526649

3. Sawale V, Dangre P, Dhabarde D. Development and validation of RP-HPLC method for the simultaneous estimation of olmesartan medoxomil and chlorthalidone in tablet dosage form. International Journal of Pharmacy and Pharmaceutical Sciences 2015; 7(5): 266-269.

4. Savale S, Mahajan H. UV Spectrophotometric Method Development and Validation for Quantitative Estimation of Diclofenac Sodium. Asian Journal of Biomaterial Research 2017; 3(2): 40-43.

5. Schiborr C, Eckert P, Rimbach G, Frank J. A validated method for the quantification of curcumin in plasma and brain tissue by fast narrow-bore high-performance liquid chromatography with fluorescence detection. Anal Bioanal Chem.2010; 397: 1917-1925. CrossRef

6. Sawale V, Dangre P, Dhabarde D. Development and validation of RP-HPLC method for the simultaneous estimation of olmesartan medoxomil and chlorthalidone in tablet dosage form. International Journal of Pharmacy and Pharmaceutical Sciences 2015; 7(5): 266-269. 